\title{
Relações entre religiosidade e saúde mental em imigrantes: implicações para a prática psi
}

\author{
Marta Helena de Freitas - Universidade Católica de Brasília, Brasília/DF, Brasil.
}

\begin{abstract}
Resumo
Este artigo visa discutir possíveis relações entre religiosidade e saúde mental de imigrantes e suas implicações para a prática clínica de psiquiatras e psicólogos que os atendem em serviços especializados. Apresenta uma síntese analítica das principais questões apontadas na literatura concernente às relações entre religiosidade e saúde mental, articulando-as com a realidade específica do imigrante, bem como apontando a relevância desta questão no contexto contemporâneo e do modo como é percebido e elaborado pelos profissionais do campo psi. Conclui-se pela necessidade de mais pesquisas sobre o assunto, cuja discussão é praticamente inexistente ao longo da formação destes profissionais.

Palavras-chave: Religiosidade; Saúde mental; Imigração; Prática psiquiátrica; Prática psicológica.
\end{abstract}

\section{Relationships between immigrants' religiosity and mental health: implications for psychiatric and psychological care}

\begin{abstract}
This paper aims to discuss the relationships between immigrants' religiosity and mental health and its implications for clinical practice of psychiatrists and psychologists who attend them in specialized services. It presents an analytical summary of the main issues raised in the literature concerning the relationships between religiosity and mental health, articulating them with immigrant's specific reality, as well as pointing to the importance of this issue in contemporary context and how it is perceived and drawn up by professionals of the PSY field. It concludes that more research is needed on the subject, whose discussion is largely absent throughout the training of PSY professionals. Keywords: Religiosity; Mental health; Immigration; Psychiatric care; Psychological care.
\end{abstract}

\section{Relaciones entre religiosidad y salud mental de los inmigrantes: implicaciones para la práctica psi}

\begin{abstract}
Resumen
En esta ponencia se analizan las posibles relaciones entre religiosidad y salud mental de los inmigrantes y de sus implicaciones para la práctica clínica de los psiquiatras y psicólogos que desempeñan servicios especializados. Presenta un resumen analítico de las principales cuestiones planteadas en la literatura sobre la relación entre religiosidad y salud mental, su vinculación a la realidad específica de los inmigrantes, así como señala la importancia de esta cuestión en el contexto actual y cómo es percibido por profesionales del PSI campo. Se concluye por la necesidad de más investigación sobre el tema, cuya discusión es prácticamente inexistente a lo largo de su formación.

Palabras clave: Religiosidad; Salud; Inmigración; Práctica psiquiátrica; Práctica psicológica.
\end{abstract}

Segundo a Organização Internacional para a Migração (IOM, 2011), no mundo todo, atualmente, uma em cada 35 pessoas é imigrante. Esta realidade é plena de implicações não apenas políticas, econômicas e socioculturais, mas também psicológicas, tanto sobre quem emigra quanto sobre quem recebe imigrantes em seus próprios países, dentre esses os profissionais de saúde mental que os atendem em serviços especializados.

Por outro lado, os temas da migração e da religião se encontram e se relacionam mutuamente no âmago da identidade dos povos e de suas respectivas organizações, como o demonstra a história do mundo, do Brasil e de sua própria capital, Brasília, conhecida por abrigar comunidades religiosas fundadas por migrantes do interior do país. No âmbito mundial, registram-se diversos impactos causados pela migração e pelo respectivo confronto do migrante com culturas totalmente diferentes e, dentro dessas, possíveis conflitos entre as normas, os valores, as línguas e as próprias religiões. Enquanto os meios de comunicação divulgam os conflitos no nível macro político, econômico e social, podemos supor outros que se passam no plano pessoal e relacional, dentre os quais aqueles relacionados às diferenças dos modelos explicativos de doença e saúde, dados pela própria cultura e crença religiosa do imigrante e as teorias e os valores nos quais se ancoram os profissionais de saúde mental que os assistem em serviços especializados. Isso fica ilustrado, por exemplo, nos depoimentos encontrados ao longo da obra organizada por Drozdek e Wilson (2007) ou nas considerações apontadas por Neubern (2012) e Freitas (2012).

Visando contribuir com um assunto tão pouco discutido na prática psi, especialmente no Brasil, e praticamente sequer mencionado na maioria dos cursos de formação profissional, este artigo assim se organiza: inicialmente apresenta algumas considerações epistemológicas e esclarecimentos conceituais sobre o tema abordado; a seguir apresenta uma síntese das principais questões apontadas na literatura concernente às relações entre religiosidade e saúde mental, articulando-as com a realidade específica do imigrante; e, por último, busca-se discutir as percepções dos profissionais psi e respectivas implicações para sua prática clínica, seguidas de algumas considerações finais. 


\section{Observações epistemológicas e esclarecimentos conceituais}

O fenômeno religioso comporta uma ambiguidade radical: ao mesmo tempo que tem uma singularidade bastante reconhecível e específica, tende a se apresentar de inúmeras maneiras nas experiências concretas dos indivíduos, das sociedades e das culturas. Tal ambiguidade reflete-se inclusive semanticamente, nos inúmeros termos empregados em psicologia da religião e na dificuldade de um consenso conceitual entre os autores, em relação à maioria deles. Mas isso não pode ser motivo para deixar-se de abordá-lo, pois reflete também a riqueza da tensão constitutiva do ser humano, cujas experiências mais significativas não se deixam aprisionar por nenhum discurso pronto $\mathrm{e}$ acabado - o que inclusive tem levado à conclusão de que religiosidade é algo que se compreende bem até o momento em que se resolve defini-la (Baltazar, 2003). Portanto, embora seja prudente iniciar estas reflexões com uma série de distinções e explanações prévias sobre o modo de compreender e abordar o fenômeno religioso, é preciso cuidar também para que tais esclarecimentos epistemológicos e conceituais não esvaziem o próprio fenômeno, a ponto de fazer com que se o perca completamente de vista.

Posto este desafio, pretende-se evitar qualquer definição absolutista, dada por doutrinas religiosas específicas ou por alguma teoria reducionista da experiência fundamental em causa. Assume-se, juntamente com Vergote (2001), o princípio fenomenológico da "exclusão metodológica do transcendente", reportando então, com o termo religiosidade, àquelas disposições humanas que levam a pessoa à capacidade - ou ausência dela - de experimentar fenômenos religiosos. Tais disposições podem ancorar-se em aspectos de ordem pessoal, social e cultural, mas são, via de regra, remetidas a uma dimensão transcendente (Deus, Sagrado, Divino, dentre outros), cuja realidade ou irrealidade ontológica não pode e não deve ser objeto de estudos da psicologia. Em vez disso, volta-se para a própria experiência humana, atentando-se para o modo como é vivida e relatada por cada sujeito e acolhendo-a no seu modo genuíno de existir e de expressar/manifestar-se no mundo circundante.

Com relação ao termo religião, desde estudos pioneiros em psicologia (James (1902/1995), houve a preocupação em distinguir vivência religiosa pessoal de religião institucional. Atualmente, costuma-se empregar o termo "religiosidade" para referir-se à experiência pessoal, subjetiva e singular no modo de vivenciar o fenômeno religioso. Quanto à expressão "religião institucional" ou "sistema religioso", o sentido contemporâneo mais frequentemente considerado quando de seu uso fica ilustrado numa feliz síntese de Amatuzzi (2001, p. 32): organização externa, mais ou menos coerente, de crenças, valores, mitos e ritos que giram em torno de um enfoque da questão do último, e que frequentemente corresponde à existência bistórica de um corpo social hierarquizado ao qual algumas pessoas dão a sua adesão.

A referida distinção, de cunho estritamente didático, tem o intuito de favorecer a comunicação, evitando-se rasurar a interação entre aspectos singulares do indivíduo e aspectos sociais e históricos que também lhe constituem, caracterizam o contexto onde está inserido e para o qual os primeiros se dirigem.

Também o fenômeno da migração e seus derivados - emigração e imigração - são bastante complexos, remetendo a alguma variedade de conceituação. Para os propósitos deste ensaio, considera-se a migração como o deslocamento, temporário ou definitivo, que implica em mudança de residência de um país para outro ou, dentro de um mesmo país, de uma região para outra (IBGE, 1969). O emigrante é aquele que deixa seu país ou região e assim é chamado por aqueles que permaneceram em seu local de origem. O imigrante, por sua vez, pode ser o mesmo indivíduo, mas agora protagonizado por aqueles que se situam no novo local em que estabeleceu a nova residência. Para o campo psi, esta questão é repleta de implicações, pois deixar o local de origem pode levar ao distanciamento das próprias raízes, e estabelecer-se em nova terra implica profunda mobilização psíquica e emocional. No título deste artigo, optamos pelo termo imigrante, tendo como parâmetro o local onde se situam os profissionais de saúde que vão atendê-lo nos serviços de saúde mental. Ao longo do texto, entretanto, eventualmente será empregado o termo migrante ou migração, quando o foco é o ato de mudar-se de um lugar para outro.

\section{Religiosidade e saúde mental: o caso do imigrante}

$\mathrm{Na}$ tumultuada história da psiquiatria (Koenig, 2000), registram-se momentos em que saúde mental e religião eram definidas de modo intrinsecamente conectados, encaminhando-se, aos poucos, para uma separação radical no discurso médico, ao ponto de, agora, multiplicarem-se, no mundo inteiro estudos tentando resgatar suas possíveis conexões. As controvérsias sobre tais conexões giram em torno de diversas questões, dentre elas: existiriam ou não correlações entre religiosidade e doença ou saúde mental?; seriam estas correlações positivas ou negativas?; estariam tais correlações em dependência de que variáveis: individuais, coletivas ou de ordem transcendente?; seria possível ou não investigar cientificamente esta questão?; no caso de ser possível, que metodologias seriam mais adequadas e/ou confiáveis: qualitativas, epidemiológicas ou mistas?

A partir de uma síntese do quadro histórico e contemporâneo sobre estudos neste campo, elaborada 
por Dalgalarrondo (2008), pode-se apreender que a natureza das respostas oferecidas para cada uma das questões apontadas anteriormente estará sempre em dependência do modo como o estudioso se dirige ao tema, o qual, por sua vez, vem associado a vários outros fatores: seu quadro teórico de fundo e a filosofia de homem a ele subjacente, seus antecedentes e atuais contextos familiares, sociais, históricos e culturais e, por que não dizer também, sua própria personalidade, incluindo-se aí o fato de ser ou não uma pessoa religiosa.

Ocorre que tanto as noções como as experiências de doença/saúde mental e de religiosidade, e mesmo as das possíveis relações entre elas, surgem em contextos socioculturais e pessoais bastante específicos e comportam um sentido fundamental, que deve também ser objeto de atenção do profissional em saúde mental. Assim, por exemplo, nas palavras de Dalgalarrondo (2006, p. 178): "é de se esperar que as diferentes formas de religiosidade, em distintos contextos sociais e culturais, tenham significações e implicações diferenciadas para a vida das pessoas, sua subjetividade e saúde mental". E o modo como se expressam essas significações pode ou não ser traduzido como sinais e sintomas, nos moldes em que as define o discurso médico ou o discurso do leigo, pois passa também por aspectos relacionados às diferenças de linguagens e pelo sentido que adquirem quando vinculado a um ou outro ordenamento social. Desse modo, as particularidades do discurso, ao serem consideradas, podem demonstrar a maior ou menor riqueza de cada experiência de vida ou de cada sinal e sintoma da doença mental em sua relação com a ordem social vigente (Baltazar, 2003). E certamente isso se torna ainda mais relevante quando se trata da saúde mental do imigrante, cuja procedência, em geral, guarda significativa alteridade em relação ao contexto para o qual migrou.

Inúmeras pesquisas empíricas têm sido realizadas nas duas últimas décadas, com o intuito de investigar as relações entre religiosidade e saúde mental e qualidade de vida, conforme diversos levantamentos da literatura (Dalgalarrondo, 2007; Koenig, McCullough \& Larson, 2001; Moreira-Almeida, Neto \& Koenig, 2006; Panzini, Rocha, Banderia \& Feck, 2007), havendo evidências de que essas relações podem ser tanto negativas quanto positivas, mas com forte predominância de uma correlação positiva entre envolvimento religioso e bemestar psicológico e ausência de sintomas psicopatológicos, especialmente em situações estressoras. Embora haja significativas diferenças entre abordagens teóricas quanto ao modo de explicar tais associações, supõe-se que, do ponto de vista psicológico, o mais provável é que ocorra uma espécie de sinergia de fatores que favoreçam essa associação positiva entre religiosidade e saúde mental, dentre eles: a rede social permitida pelo contexto em que ocorre o compartilhamento da religiosidade com pessoas de mesma fé; o sentido existencial propiciado pelo sistema de crenças e pela esperança que a fé propicia; o incentivo a hábitos de vida mais saudáveis encontrado no sistema religioso ao qual a pessoa se vincula; o sentimento de pertença experimentado pelo indivíduo, dado tanto pelo grupo religioso quanto pelo sentimento de filiação a um ser supremo.

Uma limitação dos estudos epidemiológicos e psicométricos é que eles acabam abordando a religiosidade como algo homogêneo e universal (Dalgalarrondo, 2006), enquanto os seus resultados são também de ordem abstrata, não se referindo a nenhum caso em particular, mas apenas a uma informação de cunho estatístico e de ordem genérica, deixando ao largo a dimensão propriamente humana: o phatos enquanto disposição fundamental de cada um, inserido num contexto familiar e sociocultural que lhe é próprio e particular, ou seja, o seu modo de ser e o seu processo de devenir (vir a ser), aparentar, dever e pensar junto ao mundo circundante (Martins, 2005; 2007). Além disso, a própria natureza das respostas dadas a um questionário ou a uma escala certamente passa pelo modo como o sujeito elabora cada um desses quatro verbos em seu momento de vida ou no contexto em que foi chamado a participar de uma pesquisa desta natureza. Portanto, é preciso olhar com cuidado resultados assim obtidos e buscar complementá-los com estudos que permitam um olhar mais apurado do ponto de vista clínico e fenomenológico. Isso permitiria atender melhor às duas áreas de investigação que, segundo Moreira-Almeida, Neto e Koenig (2006), ainda necessitam de maior investimento para compreender melhor as associações geralmente encontradas para o binômio religiosidade-saúde mental: a) estudo dos fatores mediadores dessa associação e b) implicação desses conhecimentos para a prática clínica.

No contexto de uma sociedade globalizada e dos grandes dilemas acerca da saúde universal que a acompanham (Periago \& Stepke, 2006-2007), multiplicam-se estudos acerca dos efeitos da migração sobre o bem-estar físico e mental das pessoas, apontando uma série de fatores que colocam o imigrante em situação de vulnerabilidade. Não apenas nos casos da migração forçada, por motivos políticos, ideológicos/religiosos ou econômicos, onde o problema é ainda mais grave, mas também nos casos da migração voluntária. E, ainda, não apenas em relação à migração malsucedida, cujo sofrimento tende a ser acentuado, mas também em relação àquela bemsucedida, pois em ambos os casos estarão presentes pelo menos dois processos psicológicos fundamentais: a elaboração da perda e o fenômeno da aculturação. Em relação ao primeiro processo, pode-se relacionar pelo menos oito elementos significativos de perda: a separação da família e dos amigos, da língua materna, da cultura, da casa, da posição social, do contato com o grupo étnico e religioso e da própria identidade (Cunha, 2007). Em relação ao segundo, que não se 
apresenta separado do primeiro, também uma série de encontros e desencontros culturais e relacionais coloca em xeque toda uma articulação de sentido da vida, levando a complexos processos de manutenção, rejeição e negociação de vários fatores que se relacionam à saúde mental. Identificado ou identificando-se como alguém que está simultaneamente dentro e fora do novo contexto sociocultural para o qual migrou (Berry, 2004), como também numa situação paradoxalmente provisória e definida nessa nova condição (Sayad, 1998), o imigrante experimenta uma série de contradições próprias da posição que ocupa. Segundo Berry (2004, p. 42), trata-se de

uma temática que muda, desconcerta e transforma identidades, relações entre homens e mulheres, concep̧çôes de mundo, valores, atitudes, relações inter-geracionais, redes sociais, hábitos alimentares, enfim, fatores presentes em toda realidade humana e que precisam ser compreendidos para que o contato entre culturas possa significar uma ampliação de possibilidades de vida e valorização dessas possibilidades.

É nessa direção, portanto, a da valorização de possibilidades para a saúde mental do imigrante, que se mostra relevante refletir sobre o papel da religiosidade nesse processo e o modo como é percebido pelos profissionais da área.

Estudos pautados em diferentes concepções teóricas realizados com os próprios imigrantes (Alves \& Ribeiro, 2002; Maalouf, 2005; Sarriera, Oliveira, Hofstaetter \& Hermel, 2005; Xavier, 2007) têm apontado importante papel desempenhado pela fé e pelas tradições religiosas em seu cotidiano. Em muitos casos, o próprio processo de migrar pode ser interpretado por seu atores como obedecendo a um impositivo maior, de ordem espiritual, legitimando a mudança e, ao mesmo tempo, oferecendo uma resposta na busca de sentido para o mesmo (Alves \& Ribeiro, 2002; Maalouf, 2005). Outras vezes, da fé inabalável em Deus provém confiança e segurança de que "tudo vai dar certo", conforme é relatado por alguns imigrantes entrevistados por Xavier (2007), em sua investigação dos processos identitários de brasileiros em Portugal, e também por imigrantes provenientes da Rússia, Líbano e Palestina vivendo em São Paulo, entrevistados por Maalouf (2005), para os quais a fé religiosa permitiu que a esperança não ficasse adoecida, mesmo nos momentos mais difíceis do processo de perda e aculturação.

Outro aspecto importante, conforme encontrado por Alves e Ribeiro (2002), ao investigar as condições de vida dos imigrantes brasileiros vivendo no sul da Flórida, EUA, é o suporte dados pelos sistemas religiosos, por meio de suas igrejas ou centros, às diversas carências e necessidades dos imigrantes, prestando diversos tipos de serviços e gerando efeitos concretos nas suas trajetórias individuais e familiares, incluindo-se aí conquistas de cunho emocional - "vida em comunidade, solidariedade, paz, atenção e acolhimento" - e espiritual - "experiência pessoal com o que dá verdadeiro sentido à vida e a projeta para além dos limites que os problemas impõem" (Alvez \& Ribeiro, 2002, p. 32).

É de se esperar que, num contexto de atenção psiquiátrica, os profissionais se deparem com outras questões mais complexas, onde, por exemplo, nos relatos de seus pacientes imigrantes acerca de seus próprios sofrimentos e respectivas atribuições de sentido, identifiquem construções que poderiam ser interpretadas como delirantes, dentre outras modalidades sintomáticas típicas de quadros descritos pelo jargão psiquiátrico. Esse tipo de preocupação tem levado ao desenvolvimento de toda uma literatura psiquiátrica e interdisciplinar que procura discutir a questão do diagnóstico diferencial entre psicopatologia e experiência religiosa ou espiritual, como ilustram alguns trabalhos britânicos (Jackson \& Fulford, 1997; 2002; Brett, 2002; Marzanski \& Bratton, 2002; Sykes, 2002). Ou, ainda, levado a estudos acerca de como as diferentes condições psiquiátricas - esquizofrenia, desordens maníaco-depressivas, ansiedade, depressão, somatização e dissociações - podem ser afetadas e analisadas a partir das diferentes influências culturais e religiosas, como encontrado em Loewenthal (2007).

O mais importante a ressaltar, entretanto, é que as experiências em geral descritas como psicopatológicas, por sua vez, interagem com diversos outros elementos próprios do contexto migratório, onde a religiosidade estará implicada: seja oferecendo sentimento de pertença ou sentido para as próprias vicissitudes no decorrer dos processos de aculturação em sua nova condição de imigrante; seja encontrando suporte por meio da vinculação a grupos e instituições religiosas, que podem trazer recursos concretos nesse novo contexto, mas também, muitas vezes, exercer um poder de tutela sobre seus próprios adeptos; seja apresentando-se como motivo de conflitos pessoais e relacionais no confronto entre a religião tradicional do imigrante e os valores da nova civilização onde este se vê, agora, inserido; seja, ainda, via fenômeno da conversão ou da emergência de fundadores de novas seitas, fato muito comum onde convive um grande contingente de pessoas provenientes de diferentes culturas. A esse respeito, vale citar uma bela frase enunciada por Menezes (2007, p. 109): “A migração abandona os valores que foram formadores, mas ao mesmo tempo os carrega como tropa de choque para a nova situação”.

\section{Religiosidade e saúde mental de imigrantes: percepção de profissionais de saúde}

$\mathrm{Na}$ psicologia e na psiquiatria, durante décadas consecutivas, ao longo do século $\mathrm{XX}$, predominaram modelos em que o tema religioso foi visto com suspeitas, desconfiança e ceticismo. Mais recentemente, 
esses modelos estão sendo revistos, repensados, contextualizados, na medida em que, diante dos muitos desafios postos pela complexidade do mundo contemporâneo, eles também se mostraram falhos, insuficientes, questionáveis.

Em pesquisa realizada num hospital do Rio de Janeiro, Baltazar (2003) constatou que os próprios profissionais sinalizam a necessidade de maior aprofundamento na compreensão das relações entre crença religiosa e saúde mental. De um modo geral, reconhecem tanto ser frequente a presença do discurso religioso nos espaços de atendimento e tratamento em saúde mental, como também a tensão pessoal e relacional que o tema suscita. Por outro lado, em seu discurso sobre as relações entre a experiência religiosa e a saúde mental, prevalece a sobreposição do "mundo natural" sobre o "mundo sobrenatural" e suas práticas se sustentam num modelo de indivíduo moderno autônomo, livre e responsável pelas suas ações e também pelo seu tratamento, refletindo um dos princípios decorrentes da reforma psiquiátrica: o de permitir aos pacientes o alcance de maior autonomia em sua vida.

Entretanto, na mesma pesquisa referida acima, a autora constatou certa dificuldade dos profissionais em tomarem o discurso e a atitude religiosa de seus pacientes como possuidores de um significado positivo sobre a saúde mental dos mesmos; em especial tais discursos e atitudes os levaram à busca de modelos destoantes do tratamento psiquiátrico e psicológico institucionalmente recebido. Por isso, tendem a não considerar as instituições religiosas como possíveis parceiras na inserção social dos pacientes. Como decorrência disso, ao comparar o discurso dos pacientes com o discurso dos profissionais, a autora constatou um distanciamento entre o projeto terapêutico tecnicamente proposto pela instituição oficial de saúde mental e o teor da experiência religiosa dos usuários, traduzindo "a impossibilidade dos profissionais em reconhecerem que a interpretação e o tratamento que propõem podem não ser os únicos e os mais indicados para alguns pacientes e que, por isso, estes também podem recorrer a 'terapêuticas religiosas" (Baltazar, 2003, p. 117). Conclui haver pouco diálogo entre os serviços voltados para a assistência em saúde mental e as instituições religiosas comunitárias, o que acaba reforçando mitos, preconceitos, malentendidos e desconhecimentos recíprocos. Assim, por exemplo, enquanto tais instituições tendem a ser vistas como fundamentalistas e autoritárias pelos profissionais de saúde mental, estes tendem a ser considerados pelas primeiras como arrogantes e onipotentes em suas práticas.

Já numa coletânea organizada por Drozdek e Wilson (2007), podem-se ler diversos relatos de experiência de profissionais de saúde mental no atendimento a imigrantes vítimas de eventos traumáticos que foram confrontadas com modelos de tratamento que diferiam daqueles com os quais estavam acostumadas e que julgavam trazer benefícios para o seu bem-estar, em especial aqueles dados pelas suas crenças e valores religiosos. Em tais descrições fica evidente que não apenas os imigrantes enfrentam dilemas quando procuram ajuda nos serviços de saúde mental, mas também os próprios profissionais que os ajudam, pois que foram ensinados a tratar os pacientes baseados em suas próprias tradições e visões de mundo e estas são culturalmente dependentes.

Como exemplo do exposto acima, num dos capítulos da referida obra, Wilson (2007) oferece uma perspectiva não ortodoxa de tratamento intercultural, chegando a descrever, inclusive, suas próprias experiências de ter sido submetido a rituais tradicionais de tratamento que eram típicos das culturas de seus próprios pacientes. A partir dessas experiências, discute sobre os componentes universais de tratamento e os que são culturalmente específicos dos rituais de cura, lembrando aos terapeutas a importância de se aprender com o próprio paciente.

Dois outros autores de capítulos da mesma coletânea, Guzder (2007) e Tang (2007), por sua vez, discutem a combinação de métodos ocidentais e não ocidentais no tratamento do trauma e sobre a importância, para terapeutas e pacientes, de se conscientizarem sobre as limitações da psiquiatria. Nessa mesma linha, Drozdek (2007) também apresenta uma maneira culturalmente sensível de realizar a psicoterapia psicodinâmica do trauma, utilizando um modelo de tratamento por ela intitulado de biopsicossocial. No seu conjunto, esses e outros trabalhos da referida obra mostram o quanto as terapias transculturais confrontam terapeutas com uma complexa gama de inter-relações próprias dos diferentes contextos culturais e políticos, as quais devem ser por eles consideradas e compreendidas.

A radical diferença encontrada entre a postura dos profissionais entrevistados na pesquisa de Baltazar (2003) e a dos que relatam suas experiências na obra organizada por Drozdek e Wilson (2007) mostra que os discursos neste campo não são, de modo algum, homogêneos e que estão a depender de inúmeras outras variáveis próprias do atual contexto de transição de paradigmas em todos os setores do conhecimento humano e suas respectivas práticas, em especial no campo da saúde mental. A migração e seus importantes impactos - políticos, econômicos, sociais e existenciais - no mundo todo são, sem dúvida, uma das principais.

\section{Considerações finais}

O desafio do encontro entre o profissional psi e o usuário do serviço de saúde mental, num contexto marcado por diferenças culturais, como é o caso do atendimento ao imigrante, pode se manifestar em relação a vários aspectos, indo desde critérios empregados para diagnóstico e prognóstico, passando 
pela natureza do vínculo terapeuta-paciente, culminando na característica dos processos de comunicação entre ambos. Em todos eles, pode-se levantar o problema da baixa conversibilidade entre a ordem da racionalidade própria da psiquiatria e da psicologia e aquela dada pela vivência pessoal e cultural do imigrante, incluindo-se aí a experiência religiosa. A partir disso, muitas questões podem ser levantadas, no que tange ao primeiro polo, no contexto do atendimento à saúde mental do paciente imigrante: Qual seria a percepção do próprio profissional de saúde no âmbito desse contexto? Que relações ele estabelece entre as duas ordens de saber - a da medicina/psicologia e a da experiência religiosa? Qual o lugar dado, em seu discurso, à experiência que é vivida pelo seu paciente como sendo de ordem religiosa? Em que e como essas questões o mobilizam enquanto profissional de saúde mental? Em que direções encaminha-se essa mobilização, seja em termos de sua concepção de saúde e doença mental, seja no que tange à condução de sua prática clínica diária com pacientes imigrantes?

A resposta a tais questões está a depender de mais pesquisas. Mas uma coisa é certa: o encontro entre dois mundos tão diferentes - o da ciência e o da religião - será mediado pelo modo como o profissional responde, para si mesmo, a cada uma delas. A radical alteridade desse encontro, no contexto de atendimento ao imigrante, remete a outros dois mundos: o da subjetividade (emoções, sentimentos, pensamentos, conhecimentos, valores...) de quem trata, sob o prisma da racionalidade científica, e o da subjetividade de quem sofre, sob o prisma de sua própria identidade pessoal e cultural - incluindo-se sua religiosidade - na busca de alívio ou de um sentido para seu sofrimento.

\section{Referências}

Alves, J. C. S. \& Ribeiro, L. (2002). Migração, religião e transnacionalismo: o caso dos brasileiros no sul da Flórida. Rio de Janeiro: Universidade Federal Rural do Rio de Janeiro e ISER. Disponível em http://scholar.google.com/scholar?q=MIgra $\% \mathrm{C} 3$ $\% \mathrm{~A} 7 \% \mathrm{C} 3 \% \mathrm{~A} 3 \mathrm{o}+$ religi $\% \mathrm{C} 3 \% \mathrm{~A} 3 \mathrm{o}+\mathrm{e}+$ transnacio nalismo\&hl $=$ en\&as_sdt $=0 \&$ as_vis $=1 \&$ oi $=$ scholar t. Acessado em 08/fev/1011.

Amatuzzi, M. M. M. (2001). Esboço de teoria do desenvolvimento religioso. Em J. G. Paiva (Org.), Entre necessidade e desejo: diálogos da psicologia com a religião (pp. 25-51). São Paulo: Loyola.

Baltazar, D. V. S. (2003). Crenças religiosas no contexto dos projetos terapêuticos em saúde mental: impasse ou possibilidade? (Dissertação de Mestrado). Rio de Janeiro: Fundação Oswaldo Cruz - Escola Nacional de Saúde Pública.

Berry, J. W. (2004). Migração, aculturação e adaptação. Em S. D. DeBiaggi \& G. J. Paiva (Orgs.).
Psicologia, E/Imigração e Cultura (pp. 29-45). São Paulo: Casa do Psicólogo.

Brett, C. (2002). Psychotic and mystical states of being: connections and distinctions. Philosophy, Psychiatry \& Psychology, 9(4), 321-341.

Cunha, M. J. C. (2007). Migração e identidade: olhares sobre o tema. São Paulo: Centauro.

Dalgalarrondo, P. (2006). Relações entre duas dimensões fundamentais da vida: saúde mental e religião. Revista Brasileira de Psiquiatria. 28(3), 177178.

Dalgalarrondo, P. (2007). Estudos sobre religião e saúde mental realizados no Brasil: histórico e perspectivas atuais. Revista de Psiquiatria Clínica, 34(1), 25-33.

Dalgalarrondo, P. (2008). Religião, psicopatologia e saúde mental. Porto Alegre: Artmed.

Drozdek, B. (2007). The story of Alex, an American man who encounters the Evil every day. Em B. Drozdek \& J. P. Wilson (Eds.), Voices of trauma: treating psychological trauma across cultures (pp. 151174). Nova Iorque: Springer.

Drozdek, B. \& Wilson, J. P. (Eds.) (2007). Voices of trauma: treating psychological trauma across Cultures. Nova Iorque: Springer.

Freitas, M. H. (2012). Religiosidade e saúde mental em imigrantes: a percepção de psiquiatras e psicólogos ingleses e brasileiros. Em M. H. Freitas, G. J. Paiva \& C. Moraes, Psicologia da religião no mundo ocidental contemporâneo: desafios da Interdisciplinaridade (pp. 257-273). Brasilia: Universa.

Guzder, J. (2007). Fourteen Djinns Migrate Across the Ocean. Em B. Drozdek \& J. P. Wilson (Eds.), Voices of trauma: treating psychological trauma across cultures (pp. 105-126). Nova Iorque: Springer.

IBGE (1969). Dicionário demográfico multilíngue. Versão brasileira. Rio de Janeiro.

IOM - International Organization for Migration (2011). About migration. Disponível em http://www.iom.int/jahia/Jahia/aboutmigration/lang/en. Acessado em 23/jan/2011.

Jackson, M. C. \& Fulford, K. W. M. (1997). Spiritual experience and psychopathology. Philosophy, Psychiatry \& Psychology, 9(1), 2-26.

Jackson, M. C. \& Fulford, K. W. M. (2002). Psychosis good and bad: values-based practice and the distinction between pathological and nonpathological forms of psychotic experience. Philosophy, Psychiatry \& Psychology, 9(4), 387-394. 
James, W. (1995). As variedades da experiência religiosa. São Paulo: Cultrix. (Publicação original em 1902).

Koenig, H. G. (2000). Religion and medicine i: historical background and reasons for separation. International Journal of Psychiatry in Medicine, 30(4), 385-398.

Koenig, H. G., McCullough, M. E. \& Larson, D. B. (2001). Handbook of religion and mental bealth. Oxford/Nova Iorque: Oxford University Press.

Loewenthal, K. (2007). Religion, culture and mental health. Cambridge/Nova Iorque: Cambridge University Press.

Maalouf, J. F. (2005). O sofrimento de imigrantes: um estudo clínico sobre os efeitos do desenraizamento do self (Tese de doutorado). São Paulo: Pontifícia Universidade Católica de São Paulo - Departamento de Psicologia Clínica.

Martins, F. (2005). Psicopathologia I: prolegômenos. Belo Horizonte: PUC Minas.

Martins, F. (2007). O aparentar, o dever, o pensar e o devir: ensaios analitico-existenciais sobre figuras exemplares do cinema e da literatura. Brasília: EDU.

Marzanski, M. \& Bratton, M. (2002). Psychopathological symptoms and religious experience: A critique of Jackson and Fulford. Philosophy, Psychiatry \& Psychology, 9(4), 359-371.

Menezes, F. L. (2007). Migração: Uma perspectiva psicológica, uma leitura pós-moderna ou, simplesmente, uma visão preconceituosa. Em M. J. C. Cunha (Org.), Migração e identidade: olhares sobre o tema (pp. 95-120). São Paulo: Centauro Editora.

Moreira-Almeida, A.; Neto, F. L. \& Koenig, H. G. (2006). Religious and mental health: Revista Brasileira Psiquiatria, 28(3), 242-250.

Neubern, M. (2012). O que significa acolher a espiritualidade do outro? Considerações de uma clínica Ethnopsy. Em M. H. Freitas; G. J. Paiva \& C. Moraes. (Orgs.), Psicologia da Religião no Mundo Ocidental Contemporâneo: Desafios da Interdisciplinaridade (pp. 145-183). Brasília: Universa.
Panzini, P. G.; Rocha, N. S.; Bandeira, D. R. \& Fleck, M. P. A. (2007). Qualidade de vida e espiritualidade. Revista de Psiquiatria Clínica, 34(1), 105-115.

Periago, M. R. \& Stepke, F. L. (2006-2007). A globalização e os dilemas da saúde universal. Ethos Gubernamental: Revista do Centro para o Desenvolvimento do Pensamento Ético, 4, 103-110.

Sarriera, J. C., Oliveira, A. A. S., Hofstaetter, C. H. \& Hermel, J. S. (2005). O processo de integração de imigrantes hispano-americanos: análises e perspectivas. Psico, 36(1), 73-80.

Sayad, A. (1991/1998). A imigração. São Paulo: EDUSP.

Sykes, S. (2002). The borderlands of psychiatry and theology. Philosophy, Psychiatry \& Psychology, 9(4): 381.

Tang, C. S. K. (2007). Culturally relevant meanings and their implications on therapy for traumatic grief: lessons learned from a Chinese female client and her fortunate-teller. Em B. Drozdek \& J. P. Wilson. (Eds.), Voices of trauma: treating psychological trauma across cultures (pp. 127-154). Nova Iorque: Springer.

Vergote, A. (2001). Necessidade e desejo da religião na ótica da psicologia. Em G. J. Paiva. (Org.), Entre necessidade e desejo: diálogos da psicologia com a religião (pp. 9-24). São Paulo: Loyola.

Wilson, J. P. (2007). Reversing cultures: the wounded theaching the healess. Em B. Drozdek \& J. P. Wilson (Eds), Voices of trauma: treating psychological trauma across cultures (pp. 87-104). Nova Iorque: Springer.

Xavier, M. (2007). Redescobrindo o Brasil: Processos identitários de brasileiros vivendo em Portugal. Lisboa, Portugal: ACIDI, IP.

Recebido em 03/10/2011

Primeira Reformulação em 22/11/2012 Segunda Reformulação em 05/03/2013

Aprovado em 14/05/2013 
Nota da autora:

Agradecimento

Ao Conselho Nacional para o Desenvolvimento Científico a Tecnológico - CNPq, por meio do seu Programa de Pós-doutorado no Exterior - PDE, que financia sua permanência na Universidade de Kent, Canterbury, possibilitando o desenvolvimento do Projeto de Pesquisa "Religiosidade e saúde mental em imigrantes na percepção de profissionais de saúde mental".

Sobre a autora:

Marta Helena de Freitas é psicóloga clínica, mestre e doutora em Psicologia - IP-UnB, com pós-doutorado em Psicologia da Religião pela University of Kent at Canterbury, Inglaterra. Coordenadora do Programa de Mestrado e Doutorado em Psicologia da UCB, onde também leciona disciplinas, orienta e desenvolve pesquisas sobre religiosidade, fenomenologia, morte e psicodiagnóstico de Rorschach. Coordenadora do GT "Psicologia \& Religião" da ANPEPP.

Contato com a autora:

Sgan 916, Módulo B, Campus II, Salas A-219/A220 - Asa Norte

CEP 70790-160 - Brasília - Distrito Federal

E-mail: mhelena@ucb.br ou mhelenadefreitas@gmail.com 